\title{
DEGREE SUM EXPONENT DISTANCE ENERGY OF SOME GRAPHS
}

\author{
SUdHIR R. JOG ${ }^{1, a}$ AND JEETENDRA R. GURJAR ${ }^{1, b}$ \\ ${ }^{1}$ Department of Mathematics, Gogte Institute of Technology, Udyambag \\ Belagavi Karnataka, 590008, India, Email: ${ }^{a}$ sudhir@git.edu, \\ ${ }^{b}$ jeetendra.g8@gmail.com
}

\begin{abstract}
The degree sum exponent distance matrix $\mathcal{M} \chi_{\text {dist }}(G)$ of a graph $G$ is a square matrix whose $(i, j)^{t h}$ entry is $\left(d_{i}+d_{j}\right)^{d_{i j}}$ whenever $i \neq j$, otherwise it is zero, where $d_{i}$ is the degree of $i^{t h}$ vertex of $G$ and $d_{i j}=d\left(v_{i}, v_{j}\right)$ is distance between $v_{i}$ and $v_{j}$. In this paper, we define degree sum exponent distance energy $E \chi_{d i s t}(G)$ as sum of absolute eigenvalues of $\mathcal{M} \chi_{\text {dist }}(G)$. Also, we obtain some bounds on the degree sum exponent distance energy of some graphs and deduce direct expressions for some graphs.

Key words and Phrases: Eigenvalue, degree sum exponent distance matrix, degree sum exponent distance energy
\end{abstract}

\section{INTRODUCTION}

The concept of graph energy was introduced by I.Gutman in 1978[1] having direct correlation with the total $\pi$-electron energy of a molecule in the quantum chemistry as calculated with the Huckel molecular orbital method. Here adjacency matrix of a graph is considered. Later Laplacian energy [2, 4], signless Laplacian energy [3], were introduced. Recently several results on energy related with degree of a vertex and distance in a graph were studied such as distance energy [5],degree sum energy of some graphs [6], degree square sum polynomial of some graphs [8], degree sum energy [9], a survey on energy of graphs [7], complementary distance energy[10], degree sum distance energy [11], degree product distance energy[12],degree exponent energy[13] and degree exponent sum energy[16].

For every pair of vertices in a connected graph there are, degree associated each one of them and in addition there is distance between them (length of the

2020 Mathematics Subject Classification: 05C50, $05 \mathrm{C} 12$. Received: 29-05-2020, accepted: 12-01-2021. 
shortest path). In continuation with this, in order to upgrade, we now introduce concept of degree sum exponent distance energy of connected graph which is slight generalization of degree sum energy since if exponent is made one, it coincides with degree sum energy. The purpose of this paper is to compute the characteristic polynomial, eigenvalues and energy of the new matrix associated with graph, called degree sum exponent distance matrix, and compute bounds for degree sum exponent distance energy and obtain expressions for some standard graphs.

\section{Degree Sum Exponent Distance Energy}

Let $G$ be a connected graph of order $n$ with vertex set $V(G)=\left(v_{1}, v_{2}, \ldots, v_{n}\right)$. We denote $d\left(v_{i}\right)$ as the degree of a vertex $v_{i}$ which is the number of edges incident on it and $d_{i j}$ as the distance between two vertices $v_{i}$ and $v_{j}$, the length of the shortest path joining them. We define degree sum exponent distance matrix of $G$ as,

$$
\begin{aligned}
& \mathcal{M} \chi_{\text {dist }}(G)=\left[\chi_{i j}\right] \text { where, } \\
& \qquad \begin{aligned}
\chi_{i j} & =\left(d\left(v_{i}\right)+d\left(v_{j}\right)\right)^{d_{i j}} \text { if } i \neq j \\
& =0 \text { if } i=j
\end{aligned}
\end{aligned}
$$

Example: For graph $G$ given below,

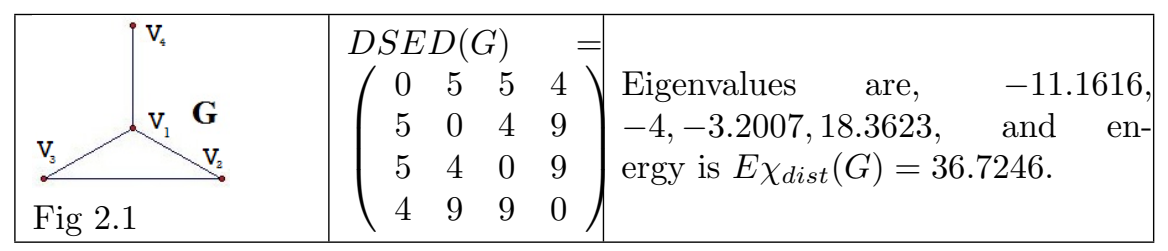

We note that,

(1) $\mathcal{M} \chi_{\text {dist }}(G)$ is real symmetric, so that the eigenvalues of $\mathcal{M} \chi_{\text {dist }}(G)$ are real. If $\alpha_{1}, \alpha_{2}, \ldots, \alpha_{n}$ are the eigenvalues of $\mathcal{M} \chi_{\text {dist }}(G)$ then, they can be arranged in a non-increasing order as $\alpha_{1} \geq \alpha_{2} \geq \ldots \geq \alpha_{n}$.

(2) $\sum_{1}^{n} \alpha_{i}=0$, since trace $\left[\mathcal{M} \chi_{\text {dist }}(G)\right]=0$.

(3) The highest exponent term corresponds to $\operatorname{diam}(\mathrm{G})$.

(4) For any r-regular graph all the entries in the matrix are in powers of $2 r$.

(5) Two non-isomorphic graphs having same order, regularity as well as diameter have same largest eigenvalue $\alpha_{1}$.

We define the degree sum exponent distance energy of a graph $G$ as,

$$
E \chi_{\text {dist }}(G)=\sum_{i=1}^{n}\left|\alpha_{i}\right| \text {. }
$$




\section{Bounds on Degree Sum Exponent Distance Energy and Eigenvalues}

In this section, we obtain some bounds on degree sum exponent distance energy and largest eigenvalue.

Lemma 3.1. Let $G$ be a graph of order $n$, then we have,

$$
\sum_{i=1}^{n} \alpha_{i}=0 \text { and } \sum_{i=1}^{n} \alpha_{i}^{2}=2 M, \text { here we define, } M=\sum_{i=1, i<j}^{n}\left(\left(d_{i}+d_{j}\right)^{d_{i j}}\right)^{2}
$$

Lemma 3.2. [15] Let $a_{1}, a_{2}, . ., a_{n}$ be non negative numbers. Then ,

$n\left[\frac{1}{n} \sum_{i=1}^{n} a_{i}-\left(\prod_{i=1}^{n} a_{i}\right)^{1 / n}\right] \leq n \sum_{i=1}^{n} a_{i}-\left(\sum_{i=1}^{n} \sqrt{a_{i}}\right)^{2} \leq n(n-1)\left[\frac{1}{n} \sum_{i=1}^{n} a_{i}-\left(\prod_{i=1}^{n} a_{i}\right)^{1 / n}\right]$

Lemma 3.3. The CauchySchwartz inequality: Let $a_{i}$ and $b_{i}, 1 \leq i \leq n$ be any real numbers, then

$$
\left(\sum_{i=1}^{n} a_{i} b_{i}\right)^{2} \leq\left(\sum_{i=1}^{n} a_{i}^{2}\right)\left(\sum_{i=1}^{n} b_{i}^{2}\right)
$$

Lemma 3.4. [17] Let $A, B, I\left(\right.$ identity matrix) and $J$ (matrix of all $1^{\prime}$ s are square matrices of same order $n$ then block determinant of order $n$,

$$
\left|A I_{n}+B\left(J_{n}-I_{n}\right)\right|=|A-B|^{n-1}|A+(n-1) B|
$$

Theorem 3.5. If $\alpha_{1}$ is the index (largest degree sum exponent distance eigenvalue) of a connected graph $G$ of order $n$, then

$$
\alpha_{1} \leq \sqrt{\frac{2 M(n-1)}{n}}
$$

where $M$ is defined above, with $d_{i j}=d\left(v_{i}, v_{j}\right)$ the distance between $v_{i}$ and $v_{j}$.

Proof. The trace of $\mathcal{M} \chi_{\text {dist }}(G)$ being zero we have

$$
\sum_{i=1}^{n} \alpha_{i}=0 \text { i.e, } \sum_{i=2}^{n} \alpha_{i}=-\alpha_{1}
$$

Further $\sum_{i=1}^{n} \alpha_{i}^{2}=\operatorname{trace} \mathcal{M} \chi_{\text {dist }}(G)^{2}=2 M$, where $M$ is as defined above. Using Lemma 3.3, with $a_{i}=1$ and $b_{i}=\alpha_{i} i=2,3 \ldots, n$ substituting we get,

$$
\left(\sum_{i=2}^{n} \alpha_{i}\right)^{2} \leq(n-1) \sum_{i=2}^{n} \alpha_{i}^{2} \leq(n-1)\left(2 M-\alpha_{1}^{2}\right)
$$

Therefore, $\left(-\alpha_{1}\right)^{2} \leq(n-1)\left(2 M-\alpha_{1}^{2}\right)$. Simplifying further,the bound for the index $\alpha_{1}$ follows.

For graph $G$ in Fig 2.1, $\alpha_{1}=-11.1616, n=4$ and $M=244$. We have, $\sqrt{\frac{2 M(n-1)}{n}}=19.1312$ 
Theorem 3.6. If $G$ is connected graph of order $n$ and $M$ is defined above, then

$$
\sqrt{2 M} \leq E \chi_{\text {dist }}(G) \leq \sqrt{2 M n}
$$

Proof. With $a_{i}=1$ and $b_{i}=\left|\alpha_{i}\right|$ and using Lemma 3.3 that is, $\left(\sum_{i=1}^{n}\left|\alpha_{i}\right|\right)^{2} \leq n \sum_{i=1}^{n}\left(\alpha_{i}\right)^{2}$. That is, $E \chi_{\text {dist }}(G)^{2} \leq 2 n M$.

Hence, $E \chi_{\text {dist }}(G) \leq \sqrt{2 M n}$.

Now for the other part,

$$
E \chi_{\text {dist }}(G)^{2}=\left(\sum_{i=1}^{n}\left|\alpha_{i}\right|\right)^{2} \geq \sum_{i=1}^{n}\left|\alpha_{i}\right|^{2}=2 M
$$

so that $E \chi_{\text {dist }}(G) \geq \sqrt{2 M}$. Combining these two,inequality follows.

For graph $G$ in Fig 2.1, we have $\sqrt{2 M}=22.09072203$ and $\sqrt{2 M n}=44.18144407$.

Theorem 3.7. If $G$ is any graph of order $n$ and $\Delta$ is the absolute value of the determinant of $\chi_{\text {dist }}(G)$ then,

$$
\sqrt{2 M+n(n-1) \Delta^{\frac{2}{n}}} \leq E \chi_{\text {dist }}(G) \leq \sqrt{2 M n}
$$

where $M$ is defined as above.

Proof. For lower bound consider,

$$
\left[E \chi_{\text {dist }}(G)\right]^{2}=\left(\sum_{i=1}^{n}\left|\alpha_{i}\right|\right)^{2}=\sum_{i=1}^{n}\left(\alpha_{i}\right)^{2}+2 \sum_{i<j}\left|\alpha_{i}\right|\left|\alpha_{j}\right|
$$

Since Arithmetic Mean (AM) $\geq$ Geometric Mean (GM) we have,

$\frac{1}{n(n-1)} \sum_{i \neq j}\left|\alpha_{i}\right|\left|\alpha_{j}\right| \geq\left(\prod_{i \neq j}\left|\alpha_{i}\right|\left|\alpha_{j}\right|\right)^{\frac{1}{n(n-1)}}=\prod_{i=1}^{n}\left(\left|\alpha_{i}\right|^{2 n-2}\right)^{\frac{1}{n(n-1)}}=\left(\prod_{i=1}^{n}\left|\alpha_{i}\right|^{\frac{2}{n}}\right)=\Delta^{\frac{2}{n}}$

using Lemma 3.2 .

therefore we have, $\prod_{i \neq j}\left|\alpha_{i}\right|\left|\alpha_{j}\right| \geq n(n-1) \Delta^{\frac{2}{n}}$.

Combining we get, $\left[E \chi_{\text {dist }}(G)\right]^{2} \geq 2 M+n(n-1) \Delta^{\frac{2}{n}}$

ie, $E \chi_{\text {dist }}(G) \geq \sqrt{2 M+n(n-1) \Delta^{\frac{2}{n}}}$

For upper bound define,

$$
\begin{gathered}
X=\sum_{i=1}^{n} \sum_{j=1}^{n}\left(\left|\alpha_{i}\right|+\left|\alpha_{j}\right|\right)^{2}=\sum_{i=1}^{n} \sum_{j=1}^{n}\left(\left|\alpha_{i}\right|^{2}+\left|\alpha_{j}\right|^{2}\right)+2\left(\sum_{i, j=1, i \neq j}^{n}\left|\alpha_{i}\right|\left|\alpha_{j}\right|\right) \\
=n \sum_{i=1}^{n}\left(\alpha_{i}\right)^{2}+n \sum_{i=1}^{n}\left(\alpha_{j}\right)^{2}-2\left(\sum_{i, j=1, i \neq j}^{n}\left|\alpha_{i}\right|\left|\alpha_{j}\right|\right) \\
=2 n M+2 n M-2\left[E \chi_{\text {dist }}(G)\right]^{2}=4 n M-2\left[E \chi_{\text {dist }}(G)\right]^{2}
\end{gathered}
$$

Since $X \geq 0$ we get $E \chi_{\text {dist }}(G) \leq \sqrt{2 M n}$ 
Combining lower bound and upper bound, we arrive at the desired result.

For the graph $G$ in Fig $2.1, \Delta=2624$ and $\sqrt{2 M+n(n-1) \Delta^{\frac{2}{n}}}=26.20495997$.

Theorem 3.8. Let $G$ be a connected $n$ vertex graph and $\Delta$ is the absolute value of the determinant of degree sum exponent distance matrix $\chi_{\text {dist }}(G)$, then

$$
\sqrt{2 M+n(n-1) \Delta^{2 / n}} \leq E \chi_{d i s t}(G) \leq \sqrt{2(n-1) M+n \Delta^{2 / n}}
$$

where $M$ is defined as above.

Proof. Let $a_{i}=\alpha_{i}^{2}, i=1,2, \ldots, n$.. Then from Lemma 3.1 and Lemma 3.2 we obtain

$n\left[\frac{1}{n} \sum_{i=1}^{n} \alpha_{i}^{2}-\left(\prod_{i=1}^{n} \alpha_{i}^{2}\right)^{1 / n}\right] \leq n \sum_{i=1}^{n} \alpha_{i}^{2}-\left(\sum_{i=1}^{n} \alpha_{i}\right)^{2} \leq n(n-1)\left[\frac{1}{n} \sum_{i=1}^{n} \alpha_{i}^{2}-\left(\prod_{i=1}^{n} \alpha_{i}^{2}\right)^{1 / n}\right]$

i.e,

$$
2 M-n \Delta^{2 / n} \leq 2 n M-\left[E \chi_{\text {dist }}(G)\right]^{2} \leq 2(n-1) M-n(n-1) \Delta^{2 / n}
$$

Thus,

$$
2 M+n(n-1) \Delta^{2 / n} \leq\left[E \chi_{\text {dist }}(G)\right]^{2} \leq 2(n-1) M+n \Delta^{2 / n}
$$

We get the desired result.

For the graph $G$ in Fig 2.1, $\sqrt{2(n-1) M+n \Delta^{2 / n}}=40.85217223$.

\section{Degree Sum Exponent Distance Energy of some graphs}

Theorem 4.1. The degree sum exponent distance energy of $K_{n}$ is, $E \chi_{\text {dist }}\left(K_{n}\right)=4(n-1)^{2}$.

Proof. The complete graph $K_{n}$ is of diameter 1 and hence every pair of vertices are at distance 1 so the degree sum exponent distance matrix of $K_{n}$ is a matrix with zero diagonal and all non diagonal entries $2(n-1)$ i.e,the degree sum exponent distance matrix of $K_{n}$ is $2(n-1)$ times the adjacency matrix of $K_{n}$. Since the adjacency energy of $K_{n}$ is $2(n-1)$, the degree sum exponent distance energy of $K_{n}$ will be $4(n-1)^{2}$.

Theorem 4.2. The degree sum exponent distance energy of $C P(n)$ is, $E \chi_{\text {dist }}(C P(n))=32 n(n-1)^{2}$.

Proof. The cocktail party graph $C P(n)$ denotes the $(2 n)$-vertex regular graph of degree $(2 n-2)$ (obtained by deleting $n$ independent edges from the complete graph $\left.K_{2 n}\right)$. Using Lemma 3.4 , where $A=(2 n-2)^{2} A\left(K_{2}\right), B=(2 n-2) J_{2 \times 2}, J$ is matrix of all 1's and $A$ is the adjacency matrix. The degree sum exponent distance polynomial of $C P(n)$ is then given by,

$\left|\alpha I-\mathcal{M} \chi_{\text {dist }}(C P(n))\right|=\left[\alpha+16(n-1)^{2}\right]^{n}[\alpha-8(n-1)(2 n-3)]^{n-1}\left[\alpha-24(n-1)^{2}\right]$ which gives, $E \chi_{\text {dist }}(C P(n))=32 n(n-1)^{2}$. 
For example, in case of $C P(4)$, eigenvalues are $-64(3$ times $), 48(2$ times $)$ and 96 giving energy, $E \chi_{\text {dist }}(C P(3))=384$.

Theorem 4.3. The degree sum exponent distance energy of crown graph $S_{n}^{0}$ is, $E \chi_{\text {dist }}\left(S_{n}^{0}\right)=16 n(n-1)^{3}$.

Proof. The crown graph is the graph obtained by removing a matching from the complete equi-bipartite graph $K_{n, n}$. So the structure of the degree product distance matrix of $S_{n}^{0}$ is,

$\mathcal{M} \chi_{\text {dist }}\left(S_{n}^{0}\right)=\left(\begin{array}{cc}A & B \\ B & A\end{array}\right)$ where $A$ is a matrix of order $n$ with zero diagonal and all non-diagonal entries as $4(n-1)^{2}$ and $B$ is the matrix of order $n$ with diagonal entry $8(n-1)^{3}$ and off diagonal entry $2(n-1)$. The eigenvalues of this matrix are given by eigenvalues of $A+B$ and eigenvalues of $A-B$ see[18].

Separately evaluating characteristic polynomials of $A+B$ and $A-B$ and then multiplying we get,degree sum exponent distance polynomial of crown graph,

$\left|\alpha I-\mathcal{M} \chi_{\text {dist }}\left(S_{n}^{0}\right)\right|=$

$\left[\alpha+2(n-1)^{2}(6 n-5)\right]\left[\alpha-2(n-1)^{2}(2 n-1)\right]\left[\alpha+2(n-1)\left(4 n^{2}-6 n+1\right)\right]^{n-1}[\alpha-$ $\left.2(n-1)\left(4 n^{2}-10 n+5\right)\right]^{n-1}$.

Adding all the absolute eigenvalues, we get the theorem.

Lemma 4.4. [14] If $a, b, c$ and $d$ are real numbers, then the determinant of the form,

$$
\left|\begin{array}{cc}
(\alpha+a) I_{n_{1}}-a J_{n_{1}} & -c J_{n_{1} \times n_{2}} \\
-d J_{n_{2} \times n_{1}} & (\alpha+b) I_{n_{2}}-b J_{n_{2}}
\end{array}\right|
$$

of order $n_{1}+n_{2}$ can be expressed in the simplified form as,

$$
(\alpha+a)^{n_{1}-1}(\alpha+b)^{n_{2}-1}\left(\left[\alpha-\left(n_{1}-1\right) a\right]\left[\alpha-\left(n_{2}-1\right) b\right]-n_{1} n_{2} c d\right)
$$

Theorem 4.5. The degree sum exponent distance energy of the complete bipartite graph $K_{m, n}$ is, $E \chi_{\text {dist }}\left(K_{m, n}\right)=8 n^{2}(m-1)+8 m^{2}(n-1)$.

Proof. In $K_{m, n}, m$ vertices have degree $n$ and $n$ vertices have degree $m$. The diameter being 2 , the structure of the degree sum exponent distance matrix is,

$$
\mathcal{M} \chi_{\text {dist }}\left(K_{m, n}\right)=\left[\begin{array}{cc}
4 n^{2} A\left(K_{m}\right) & (m+n) J_{m \times n} \\
(m+n) J_{n \times m} & 4 m^{2} A\left(K_{n}\right)
\end{array}\right]
$$

where $J$ is matrix of all 1 's and $A$ is the adjacency matrix. The degree sum exponent distance polynomial is then given by,

$$
\left|\alpha I-\mathcal{M} \chi_{\text {dist }}\left(K_{m, n}\right)\right|=\left|\begin{array}{cc}
\alpha I_{m}-4 n^{2} A\left(K_{m}\right) & -(m+n) J_{m \times n} \\
-(m+n) J_{n \times m} & \alpha I_{n}-4 m^{2} A\left(K_{n}\right)
\end{array}\right| .
$$

Using Lemma 4.4 we get the degree sum exponent distance polynomial, $\left|\alpha I-\mathcal{M} \chi_{\text {dist }}\left(K_{m, n}\right)\right|=$ $\left[\alpha+4 n^{2}\right]^{m-1}\left[\alpha+4 m^{2}\right]^{n-1}\left[\alpha^{2}-4\left(n^{2}(m-1)+m^{2}(n-1)\right) \alpha+16 m^{2} n^{2}(n-1)(m-\right.$ 
1) $\left.-(m+n)^{2} m n\right]$

The quadratic equation above has $\left[4\left(n^{2}(m-1)+m^{2}(n-1)\right]^{2}>4 \times\left[16 m^{2} n^{2}(n-\right.\right.$ 1) $\left.(m-1)-(m+n)^{2} m n\right]$ hence sum of absolute roots is $4\left(n^{2}(m-1)+m^{2}(n-1)\right)$ and on adding all absolute eigenvalues the theorem follows.

For example, in case of $K_{3,4}$, eigenvalues are -64(2times), 36(3times), 91.7702 and 144.2298 giving the energy,$E \chi_{\text {dist }}\left(K_{3,4}\right)=472$.

Corollary 4.6. The degree sum exponent distance energy of the star graph $K_{1, n}$ is, $E \chi_{\text {dist }}\left(K_{1, n}\right)=8(n-1)$.

Proof. Put $m=1$ in Theorem 4.5.

Corollary 4.7. The degree sum exponent distance energy of the equi-bipartite graph $K_{n, n}$ is, $E \chi_{\text {dist }}\left(K_{n, n}\right)=16 n^{2}(n-1)$.

Proof. Put $m=n$ in Theorem 4.5.

Theorem 4.8. If $B_{n}(n \geq 3)$ is a book graph of order $(n+2)$ with triangular pages and size $(2 n+1)$, then $E \chi_{\text {dist }}$ of $B_{n}$ is, $E \chi_{\text {dist }}\left(B_{n}\right)=36 n-28$

Proof. The book graph $B_{n}$ with triangular pages has two sets of vertices, a set with $n$ vertices of degree 2 and the remaining 2 vertices of degree $(n+1)$. The structure of the degree sum exponent distance matrix is,

$$
\mathcal{M} \chi_{\text {dist }}\left(B_{n}\right)=\left[\begin{array}{cc}
2(n+1) A\left(K_{2}\right) & (n+3) J_{2 \times n} \\
(n+3) J_{n \times 2} & 16 A\left(K_{n}\right)
\end{array}\right]
$$

where $J$ is matrix of all 1's and $A$ is the adjacency matrix. The degree sum exponent distance polynomial is then given by,

$$
\left|\alpha I-\mathcal{M} \chi_{\text {dist }}\left(B_{n}\right)\right|=\left|\begin{array}{cc}
\alpha I_{2}-2(n+1) A\left(K_{2}\right) & -(n+3) J_{2 \times n} \\
-(n+3) J_{n \times 2} & \alpha I_{n}-16 A\left(K_{n}\right)
\end{array}\right| .
$$

Using Lemma 4.4 we get the degree sum exponent distance polynomial,

$\left|\alpha I-\mathcal{M} \chi_{\text {dist }}\left(B_{n}\right)\right|=[\alpha+16]^{n-1}[\alpha+2(n+1)]\left[\alpha^{2}-2(9 n-7) \alpha+2(16(n-1)(n+\right.$ 1) $\left.\left.-n(n+3)^{2}\right)\right]$.

The quadratic equation above has $[2(9 n-7)]^{2}>4 \times 2\left(16(n-1)(n+1)-n(n+3)^{2}\right)$ hence sum of absolute roots is $2(9 n-7)$ and the theorem follows on adding all absolute eigenvalues.

Let $K_{n}-e$ and $K_{n}+e$ denote the graph obtained from complete graph $K_{n}$ by deleting an edge, adding an edge respectively. 
Theorem 4.9.

$$
\begin{aligned}
E \chi_{\text {dist }}\left(K_{n}-e\right) & =44.3606 \text { if } n=4 \\
& =4\left[(n-1)(n-3)+2(n-2)^{2}\right] \text { if } n>4
\end{aligned}
$$

Proof. The graph $K_{n}-e$ is of diameter 2 and has two vertices with distance two and remaining at distance one.

For $n=4$, using Matlab we have $E \chi_{\text {dist }}\left(K_{4}-e\right)=44.3606$

The degree sum exponent distance matrix of $K_{n}-e$ has the form,

$$
\mathcal{M} \chi_{\text {dist }}\left(K_{n}-e\right)=\left[\begin{array}{ccc}
0 & 4(n-2)^{2} & (2 n-3) J_{1 \times n-2} \\
4(n-2)^{2} & 0 & (2 n-3) J_{1 \times n-2} \\
(2 n-3) J_{n-2 \times 1} & (2 n-3) J_{n-2 \times 1} & 2(n-1) A\left(K_{n-2}\right)
\end{array}\right] \text {. }
$$

So that the degree sum exponent distance polynomial of $K_{n}-e$ is given by, $\left|\alpha I-\mathcal{M} \chi_{\text {dist }}\left(K_{n}-e\right)\right|$

$$
=\left|\begin{array}{ccc}
\alpha & -4(n-2)^{2} & -(2 n-3) J_{1 \times n-2} \\
-4(n-2)^{2} & \alpha & -(2 n-3) J_{1 \times n-2} \\
-(2 n-3) J_{n-2 \times 1} & -(2 n-3) J_{n-2 \times 1} & \alpha I_{n-2}-2(n-1) A\left(K_{n-2}\right)
\end{array}\right|
$$

Using Lemma 4.4 we get the degree sum exponent distance polynomial, $\left|\alpha I-\mathcal{M} \chi_{\text {dist }}\left(K_{n}-e\right)\right|=\left[\alpha+4(n-2)^{2}\right][\alpha+2(n-1)]^{n-3}\left[\alpha^{2}-2((n-1)(n-3)+\right.$ $\left.\left.2(n-2)^{2}\right) \alpha+2(n-2)\left(4(n-1)(n-3)(n-2)-(2 n-3)^{2}\right)\right]$ for $n>4$. Since $\left(2\left((n-1)(n-3)+2(n-2)^{2}\right)^{2}>4 \times 2(n-2)\left(4(n-1)(n-3)(n-2)-(2 n-3)^{2}\right)\right.$, the sum of absolute roots of the quadratic is $\left(2\left((n-1)(n-3)+2(n-2)^{2}\right)\right.$.

Hence the theorem.

Theorem 4.10. $E \chi_{\text {dist }}\left(K_{n}+e\right)=2(n-1)(n-2)+\left|\alpha_{1}\right|+\left|\alpha_{2}\right|+\left|\alpha_{3}\right|$, where $\alpha_{1}, \alpha_{2}$ and $\alpha_{3}$ are roots of the equation, $\left[\alpha^{3}-2(n-1)(n-2) \alpha^{2}-\left((2 n-1)^{2}(n-1)+n^{4}(n-1)+(n+1)^{2}\right) \alpha+2(n+1)(n-\right.$ $\left.1)\left((n+1)(n-2)-n^{2}(2 n-1)\right)\right]=0$.

Proof. In $K_{n}+e$ there is one vertex with degree $n$, one vertex with degree 1 and remaining $n-1$ have degree $n-1$. Thus we get the degree sum exponent distance matrix with suitable labeling as,

$$
\mathcal{M} \chi_{\text {dist }}\left(K_{n}+e\right)=\left[\begin{array}{ccc}
0 & (n+1) & (2 n-1) J_{1 \times n-1} \\
(n+1) & 0 & n^{2} J_{1 \times n-1} \\
(2 n-1) J_{n-1 \times 1} & n^{2} J_{n-1 \times 1} & 2(n-1) A\left(K_{n-1}\right)
\end{array}\right] .
$$

So that the degree sum exponent distance polynomial of $K_{n}+e$ is given by,

$$
=\left|\begin{array}{ccc}
\left|\alpha I-\mathcal{M} \chi_{\text {dist }}\left(K_{n}+e\right)\right| & & \\
\alpha & -(n+1) & -(2 n-1) J_{1 \times n-1} \\
-(n+1) & \alpha & -n^{2} J_{1 \times n-1} \\
-(2 n-1) J_{n-1 \times 1} & -n^{2} J_{n-1 \times 1} & \alpha I_{n-1}-2(n-1) A\left(K_{n-1}\right)
\end{array}\right|
$$

Using Lemma 4.4 we get the degree sum exponent distance polynomial,

$\left|\alpha I-\mathcal{M} \chi_{\text {dist }}\left(K_{n}+e\right)\right|=[\alpha+2(n-1)]^{n-2}\left[\alpha^{3}-2(n-1)(n-2) \alpha^{2}-\left((2 n-1)^{2}(n-\right.\right.$ $\left.\left.1)+n^{4}(n-1)+(n+1)^{2}\right) \alpha+2(n+1)(n-1)\left((n+1)(n-2)-n^{2}(2 n-1)\right)\right]$.

On extracting eigenvalues and taking the absolute sum, we get the theorem.

For example, in case of $K_{5}+e$, eigenvalues are $-40.5244,-8(3$ times $),-3.5991$, 68.1235 giving the energy as, $E \chi_{\text {dist }}\left(K_{5}+e\right)=136.247$. 
Definition 4.11 (Vertex Coalescence). If $G_{1}$ and $G_{2}$ are any two graphs then the graph obtained by gluing $G_{1}$ and $G_{2}$ at a point is $v$ called vertex coalescence denoted by $\mathrm{G}_{1} \mathrm{O}_{v} \mathrm{G}_{2}$.

Definition 4.12 (Edge Coalescence). If $G_{1}$ and $G_{2}$ are any two graphs then the graph obtained by merging $G_{1}$ and $G_{2}$ on an edge $e$ is called edge coalescence denoted by $\mathrm{G}_{1} \mathrm{O}_{e} G_{2}$.

Now we consider the degree sum exponent distance energy of vertex coalescence and edge coalescence of complete graphs of same order. Let $K_{n}$ be a complete graph of order $n$ then the vertex coalescence of $K_{n}$ with $K_{n}$ will be denoted by $K_{n} O_{v} K_{n}$ and the edge coalescence by $K_{n} O_{e} K_{n}$.

$K_{n} O_{v} K_{n}$ has $2 n-1$ vertices and $2 \times\left({ }^{n} C_{2}\right)$ edges whereas $K_{n} O_{e} K_{n}$ has $2 n-2$ vertices and $2 \times\left({ }^{n} C_{2}-1\right)$ edges.

Lemma 4.13. [19] Let $a$ and $b$ be two arbitrary constants, $I$ is the identity matrix and $J$ is $n \times n$ matrix whose all entries $1^{\prime}$ s. If $A=(a-b) I+b J$ then the characteristic polynomial of $A$ is, $|\lambda I-A|=[\lambda-a+b]^{n-1}[\lambda-a-(n-1) b]$.

Theorem 4.14. The degree sum exponent distance energy of the vertex coalescence of two complete graphs $K_{n}$ for $n \geq 3$ is given by,

$E \chi_{\text {dist }}\left(K_{n} O_{v} K_{n}\right)=2(n-1)\left(2 n^{2}-5 n+8\right)+2(n-1) \sqrt{\left(2 n^{2}-5 n+8\right)^{2}+18(n-1)}$.

Proof. The graph $K_{n} O_{v} K_{n}$ has two sets of vertices one at a distance 2 from each other and other at 1 , being of diameter 2 . With suitable labeling the degree sum exponent distance matrix of $K_{n} O_{v} K_{n}$ takes the form,

$$
\mathcal{M} \chi_{\text {dist }}\left(K_{n} O_{v} K_{n}\right)=\left[\begin{array}{ccc}
0 & 3(n-1) J_{1 \times n-1} & 3(n-1) J_{1 \times n-1} \\
3(n-1) J_{n-1 \times 1} & 2(n-1) A\left(K_{n-1}\right) & 4(n-1)^{2} J_{n-1 \times n-1} \\
3(n-1) J_{n-1 \times 1} & 4(n-1)^{2} J_{n-1 \times n-1} & 2(n-1) A\left(K_{n-1}\right)
\end{array}\right]
$$

So that the degree sum exponent distance polynomial of $K_{n} O_{v} K_{n}$

$\left|\alpha I-\mathcal{M} \chi_{\text {dist }}\left(K_{n} O_{v} K_{n}\right)\right|=$

$$
\left|\begin{array}{ccc}
\alpha & -3(n-1) J_{1 \times n-1} & -3(n-1) J_{1 \times n-1} \\
-3(n-1) J_{n-1 \times 1} & \alpha I_{n-1}-2(n-1) A\left(K_{n-1}\right) & -4(n-1)^{2} J_{n-1 \times n-1} \\
-3(n-1) J_{n-1 \times 1} & -4(n-1)^{2} J_{n-1 \times n-1} & \alpha I_{n-1}-2(n-1) A\left(K_{n-1}\right)
\end{array}\right|
$$

Using Lemma 4.11 we get the degree sum exponent distance polynomial,

$\left|\alpha I-\mathcal{M} \chi_{\text {dist }}\left(K_{n} O_{v} K_{n}\right)\right|=$

$\left[\alpha+2(n-1)\left(2(n-1)^{2}-n+2\right)\right][\alpha+2(n-1)]^{2 n-4}\left[\alpha^{2}-2(n-1)\left(2 n^{2}-5 n+8\right) \alpha-\right.$ $\left.18(n-1)^{3}\right]$.

On extracting eigenvalues and taking the absolute sum, we get the theorem.

For example, in case of $K_{5} O_{v} K_{5}$, eigenvalues are $-232,-8(6$ times $),-4.0555$, 284.0555 giving the energy as, $E \chi_{\text {dist }}\left(K_{5} O_{v} K_{5}\right)=568.111$.

Theorem 4.15. The degree sum exponent distance energy of the edge coalescence of two complete graphs $K_{n}$ for $n \geq 3$ is given by, $E \chi_{\text {dist }}\left(K_{n} O_{e} K_{n}\right)=4(2 n-3)+$ $4(n-1)\left(2 n^{2}-7 n+7\right)+8(n-1)(n-3)$. 
Proof. The graph $K_{n} O_{e} K_{n}$ has two sets of vertices one at a distance 2 from each other and other at 1 , being of diameter 2 . There are two vertices of degree $(2 n-3)$ and remaining $(2 n-4)$ of degree $(n-1)$. With suitable labeling the degree sum exponent distance matrix of $K_{n} O_{e} K_{n}$ takes the form,

$\mathcal{M} \chi_{\text {dist }}\left(K_{n} O_{e} K_{n}\right)=\left[\begin{array}{ccc}2(2 n-3) A\left(K_{2}\right) & (3 n-4) J_{2 \times n-2} & (3 n-4) J_{2 \times n-2} \\ (3 n-4) J_{n-2 \times 2} & 2(n-1) A\left(K_{n-2}\right) & 4(n-1)^{2} J_{n-2 \times n-2} \\ (3 n-4) J_{n-2 \times 2} & 4(n-1)^{2} J_{n-2 \times n-2} & 2(n-1) A\left(K_{n-2}\right)\end{array}\right]$

So that the degree sum exponent distance polynomial of $K_{n} O_{e} K_{n}$ is given by, $\left|\alpha I-\mathcal{M} \chi_{\text {dist }}\left(K_{n} O_{e} K_{n}\right)\right|=$

$$
\left|\begin{array}{ccc}
\alpha I_{2}-2(2 n-3) A\left(K_{2}\right) & -(3 n-4) J_{2 \times n-2} & -(3 n-4) J_{2 \times n-2} \\
-(3 n-4) J_{n-2 \times 2} & \alpha I_{n-2}-2(n-1) A\left(K_{n-2}\right) & -4(n-1)^{2} J_{n-2 \times n-2} \\
-(3 n-4) J_{n-2 \times 2} & -4(n-1)^{2} J_{n-2 \times n-2} & \alpha I_{n-2}-2(n-1) A\left(K_{n-2}\right)
\end{array}\right|
$$

So that the degree sum exponent distance polynomial of $K_{n} O_{e} K_{n}$ is given by,

$\left|\alpha I-\mathcal{M} \chi_{\text {dist }}\left(K_{n} O_{e} K_{n}\right)\right|=[\alpha+2(2 n-3)]\left[\alpha+2(n-1)\left(2 n^{2}-7 n+7\right)\right][\alpha+2(n-$ $1)]^{2 n-6}\left[\alpha^{2}-2\left(2 n^{3}-7 n^{2}+8 n-4\right) \alpha+4(2 n-3)(n-1)((2 n-1)(n-3)+2 n-2)-\right.$ $\left.4(3 n-4)^{2}(n-2)\right]$.

On extracting eigenvalues and taking the absolute sum, we get the following theorem.

For example, in case of $K_{5} O_{e} K_{5}$, eigenvalues are $-176,-14,-8(4$ times $)$, -6.7839, 215.2161 giving the energy as, $E \chi_{\text {dist }}\left(K_{5} O_{e} K_{5}\right)=444.003$.

From Theorem 4.2 and Theorem 4.3 we see that both $S_{3}^{0}$ and $C P(3)$ have same $E \chi_{\text {dist }}=384$, although $C P(3)$ has 12 edges and $S_{3}^{0}$ has 6 edges. We call such graphs as degree sum exponent distance equi-energeic.

Definition 4.16. Two non isomorphic graphs on same number of vertices are said to be degree sum exponent distance equi-energeic if they have same degree sum exponent distance energy.

\section{Conclusion}

We discussed the degree sum exponent distance energy of graphs. Also, we discussed bounds on the energy of degree sum exponent distance energy. There is scope to investigate degree sum exponent distance energy of graphs with higher diameter,trees, unicyclic graphs etc and also to construct degree sum exponent distance equi-energetic graphs. 


\section{REFERENCES}

[1] Gutman, I., "The Energy of a Graph", Ber. Math. Stat. Sekt. Forschungsz. Graz , 103 (1978), pp.1-22.

[2] Gutman, I., Zhou, B., "Laplacian energy of a graph", Linear Algebra and its appl., 414(1), (2006), 29-37.

[3] Cvetkovic, D., Rowlinson, P., and Simic, S., "Signless Laplacians of finite graphs", Linear Algebra and its Appl. 423(1) (2007), 155-171.

[4] Das, K.Ch. and Mojallal, S. Ahhad , "On energy and Laplacian energy of graphs", Elec. J. of Linear Algebra, 31 (2016), 167-186.

[5] Indulal, G., Gutman, I., and Vijaykumar, A.," On distance energy of graphs", MATCH Commun. Math. Comput. Chem 60 (2008), 461-472.

[6] Jog, S.R. and Kotambari, R., "Degree Sum Energy of Some Graphs ", Annals of Pure and Appl. Math.11(1) (2016),17-27.

7] Meenakshi, S. and Lavanya, S., "A Survey on Energy of Graphs", Annals of Pure and Appl. Math.8(2) (2014), 183-191.

[8] Basavanagoud, B. and Chitra, E., "Degree Square Sum Polynomial of some Special Graphs", Int. J. Math. And Appl.13(19) (2018), 14060-14078.

[9] Ramane, H.S., Revenkar, D.S., and Patil, J.B., "Bounds for the degree sum eigenvalues and degree sum energy of a graph",Int. J. Pure App. Sci, 6(2) (2013), 161-167.

[10] Ramane, H.S. and Nandesh, K.C., "Complementary distance spectra and complementary distance energy of line graph of regular graphs",J. Indones. Math. Soc 22(1) (2016), 2735.

[11] Jog, S.R. and Gurjar, J.R., "Degree sum distance energy of some graphs", communicated.

[12] Jog, S.R. and Gurjar, J.R., "Degree product distance energy of some graphs", Asian J. Math and Comp.Res. 24(1) (2018) 42-49.

[13] Ramane, H.S. and Shinde, S.S., "Degree exponent polynomial and degree exponent energy of graphs", Indian J. Discrete Math 2(1), (2016), 1-7.

[14] Ramane, H.S. and Shinde, S.S., "Degree exponent polynomial of graphs obtained by some graph operations", Electronics Notes in Discrete Mathematics 63 (2017), 161-168.

[15] Kober, H., "On the arithmetic and geometric means and on Holder's inequality".Proc. Amer. Math. Soc. 9 (1958), 452-459.

[16] Basavanagoud, B. and Eshwarachandra, C., "Degree exponent sum energy of a graph", Gulf J. of math. 8(1) (2020), 52-70.

[17] Grantmacher, F.R., Theory of Matrices, I Chelsea New York, 1960.

[18] Davis, P.J., Circulant Matrices, Wiley, New york, 1979.

[19] Bapat, R.B., Graphs and matrices, Springer, New York, 2010. 